\title{
La naturaleza de la capa de preparación según la visión de algunos de los principales tratadistas de la historia de la pintura
}

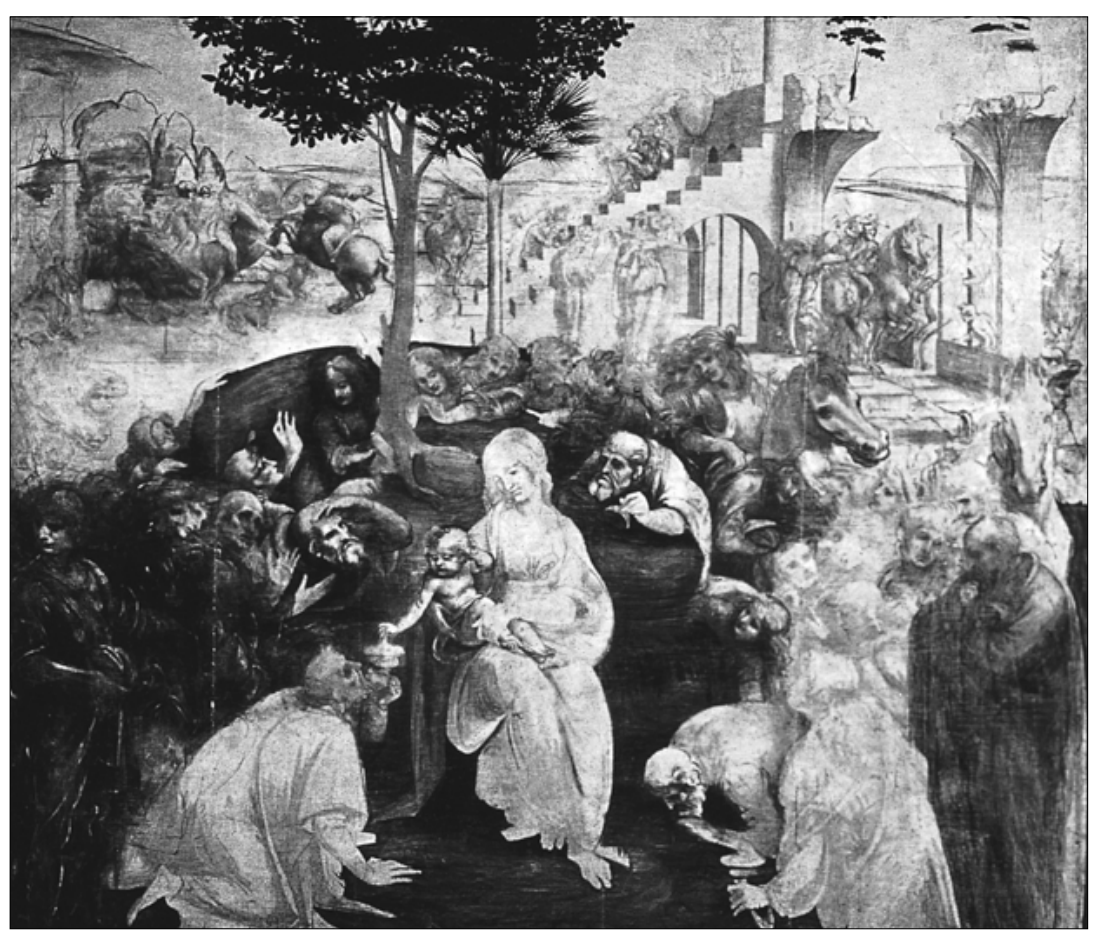

Ma José González López Jefa del Departamento de Tratamiento Instituto Andaluz del Patrimonio Histórico

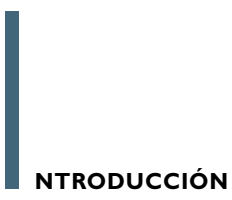

¿Qué entendemos por preparar un soporte?. Este concepto comprende un conjunto de operaciones dirigidas a rendir apto un soporte pictórico para recibir la pintura en las mejores condiciones posibles de absorción, adherencia, textura, etc. Su objetivo no es otro que disponer de una base adecuada para pintar. Incluye la aplicación de una serie de estratos que pueden ser de naturaleza diferente o cumplir funciones diversas entre sí. Normalmente abarca: tratamiento preliminar del soporte, aplicación de una capa aislante, aplicación de un fondo (comúnmente denominado preparación) e incluso la realización de una capa de imprimación, con o sin capa aislante intermedia. Como veremos en el análisis de 


\begin{tabular}{ccc|cc}
\hline & \multicolumn{3}{c}{$\begin{array}{c}\text { Cuadro } N^{\circ} \text { I: Composición del conjunto pictórico en } \\
\text { función del soporte y de la técnica pictórica }\end{array}$} \\
\hline \multicolumn{3}{c}{ TELA } \\
\hline OLEO & TEMPLE & OLEO & TEMPLE \\
\hline Soporte & Soporte & Soporte & Soporte \\
Aislante & Aislante & Aislante & Aislante \\
Imprimación & Pintura & Preparación & Preparación \\
Pintura & & $\begin{array}{c}\text { Imprimación } \\
\text { Pintura }\end{array}$ & Pintura \\
& & & & \\
\hline
\end{tabular}

los principales tratados de la Historia de la Pintura consultados, esta sucesión de estratos no siempre aparecen conjuntamente sino que su elección, naturaleza y disposición están relacionados con tres factores: soporte, técnica pictórica y época de ejecución de la obra.

La capa de preparación ha sido uno de los estratos más olvidados por los técnicos y estudiosos que han investigado la configuración material de una obra pictórica. Esta omisión, sin duda motivada por su ubicación en la pintura, ha dado como consecuencia directa, al no ser visible externamente, ser un conjunto de estratos poco estudiados, pero que por el contrario desempeñan un importante rol en la ejecución de la obra, en su conservación temporal, e incluso en su percepción estética final

El vocablo Preparación tiene un doble sentido pictóricamente hablando: Uno general, preparación del soporte pictórico, y uno específico, realización de la capa de preparación magra (capa compuesta por una sustancia de carga o relleno aglutinada en un medio de naturaleza proteica, normalmente de color blanco). No ocurre lo mismo con el término Imprimación que define una de las fases incluidas en la preparación de un soporte pictórico, nos referimos a la capa de fondo compuesta por pigmentos secativos y un aglutinante oleoso, generalmente coloreada. También nos encontramos en la terminología habitual dos criterios diferentes para definir el vocablo preparación (I), la primera de ellas se acoge a la naturaleza del aglutinante que interviene en su composición, y la segunda, al color que puede recibir este/s estrato/s. En este trabajo nos ceñiremos a la primera opción, es decir, a su naturaleza, ya que en cierto modo condiciona, hasta cierto punto, la técnica pictórica seleccionada por el artista (2).

\section{LA CAPA DE PREPARACIÓN SEGÚN ALGUNOS DE LOS PRINCIPALES TRATADISTAS DE LA HISTORIA DE LA PINTURA}

El punto de partida de este trabajo se inicia con la investigación en las fuentes literarias tradicionales, análisis de los manuscritos y tratados que han estudiado de forma directa e indirecta la preparación de un soporte pictórico. Esta investigación ha puesto de manifiesto datos suficientes para poder establecer, desde un punto de vista teórico unas serie de cuestiones de interés, técnicas y conservativas: ¿cuáles fueron los materiales más empleados en su elaboración?, ¿cómo condiciona la naturaleza de una técnica pictórica su composición, o la del soporte que le sirve de base?, ¿De qué forma se ha venido habitualmente aplicando y con qué herramientas? y por último, ¿cómo evoluciona a través de la Historia de la Pintura?

Con objeto de responder a estas dudas se ha consultado tratados y tratadistas seleccionados en base a los siguientes criterios: Época, ubicación geográfica y fundamentalmente aquellos en los que hemos encontrado referencias directas sobre este tema.

Una obra pictórica sobre madera o tela está realizada por una superposición de estratos aplicados sobre el soporte seleccionado por el artista. Las combinaciones más habitualmente detectadas las exponemos en el cuadro $n^{\circ}$ ।:

\section{TIPOS DE PREPARACIONES}

De igual forma, los principales tipos de preparaciones encontrados los podemos clasificar en tres categorías según la naturaleza del aglutinante que interviene en su composición: preparación magra, imprimación oleosa y fondo mixto.

Preparación magra: Entendiendo como tal aquellas preparaciones constituidas por una sustancia de carga y/o pigmento cubriente aglutinada en un medio acuoso. A igual que ocurre con las técnicas pictórica, el aglutinante empleado es el que condiciona su composición. Son las más antiguas, pues sabemos que eran conocidas y empleadas por los egipcios tanto en los célebres retratos de la región del Fayún (3), como en sus sarcófagos (4). A partir de entonces se utilizan en todas las épocas y estilos sin apenas modificaciones alcanzando su máximo esplendor en la Escuela Italiana del Trencento y en la Flamenca del Quinientos. Sin lugar a dudas son las bases más recomendadas para el soporte de madera y la técnica pictórica del temple en todas sus variedades con o sin aplicaciones de láminas de metal (oro o plata). Se caracterizan por ser muy absorbentes, luminosas, de fácil aplicación, rápido secado y permite obtener un acabado superficial según las necesidades del artista (liso, pulido, relieves, etc). En la literatura también las encontramos bajo las siguientes designaciones: fondo de "gesso", de creta, de caseína y de engrudo principalmente. En general son de color blanco.

Una excepción a esta regla la constituye la capa aislante de naturaleza proteica que recibe el soporte de tela como única preparación para la pintura, en este caso, al temple.

Imprimación oleosa: Entendemos aquellas preparaciones compuestas por un aglutinante de naturaleza grasa, generalmente aceites secativos o sus emulsiones, y por pigmentos cubrientes. Este tipo de fondo es 
característico del soporte de tela ya que su desarrollo está estrechamente relacionado con la técnica del óleo. En general son coloreadas y se aplican sobre el soporte previamente aislado, normalmente con un aislante de naturaleza proteica, para evitar interacciones negativas en la conservación de la obra acabada. Se deben observar unas normas muy escrupulosas en su aplicación si se desea evitar la aparición de alteraciones prematuras en la pintura, en general se recomienda el uso de pigmentos de acción secante, limitar la cantidad del aglutinante en su composición, extender con espátula en capa delgada y, no utilizar el soporte preparado de esta forma hasta su completo secado. Heraclius es el primero en describirnos este tipo de fondo para el soporte de madera empleando blanco de plomo molido con aceite de linaza (5).

Fondo mixto: Este tipo de fondo está constituido por la superposición de los dos tipos de fondos ya comentados según el siguiente esquema: preparación magra e imprimación oleosa, con o sin capa aislante intermedia. $\mathrm{Se}$ introduce en los soportes de madera a partir del $\mathrm{S}$. $\mathrm{XV}$, como una adaptación de los fondos magros tradicionales para la técnica del temple a las nuevas exigencias de la pintura al óleo, intentando reunir en su conjunto las ventajas que pueden aportar ambas técnicas. En sus inicios ambas capas son de color blanco, pero con el desarrollo de la pintura al óleo sobre tela, se colorea también la imprimación que recibe el soporte de madera. Este tipo de fondo han sido recomendados desce Vasari (6) hasta prácticamente el S. XVIII.

\section{COMPOSICIÓN MATERIAL DE LA CAPA DE PREPARACIÓN}

En la composición de estos estratos intervienen una serie de materiales básicos con mayor o menor número de aditivos, los principales materiales detectados los exponemos seguidamente en función del tipo de soporte seleccionado. (Véase cuadro $n^{\circ} 2$ ).

\section{Aglutinantes}

Se define como tal aquella sustancia empleada con fines pictóricos para unir los granos del pigmento o de la carga entre sí y permitir que estos permanezcan adheridos al soporte o revestimiento sobre el que es aplicado. Su elección está unido a varios factores entre los que destacamos: soporte seleccionado, pigmento o carga al que aglutina, empleo a que se destina (aislante, impermeabilizante) y técnica pictórica escogida por el artista.

Los aglutinantes magros empleados en la preparación de los soportes pictóricos los podemos clasificar en dos tipos según su naturaleza:

Derivados de una sustancia orgánica, básicamente: colas animales, albúmina y caseína. Están compuestos por proteínas (carbono, hidrógeno y oxígeno) provenientes del reino animal.
Cuadro $\mathrm{N}^{\circ}$ 2: Principales materiales que intervienen en la composición de las capas de preparación

\begin{tabular}{|c|c|}
\hline SOPORTE DE MADERA & SOPORTE DE TELA \\
\hline $\begin{array}{l}\text { AGLUTINANTE MAGRO: } \\
\text { - ANIMALES: } \\
\text { - COLA } \\
\text { - ALBUMINA } \\
\text { - CASEÍNA } \\
\text { AGLUTINANTE GRASO: } \\
\text { - ACEITE SECATIVO: } \\
\text { - ACEITE DE LINAZA } \\
\text { - ACEITE DE NUEZ }\end{array}$ & $\begin{array}{l}\text { AGLUTINANTE MAGRO: } \\
\text { - ANIMALES: } \\
\text { - COLA } \\
\text { - ALBUMINA } \\
\text { - CASEÍNA } \\
\text { - VEGETALES: } \\
\text { - ENGRUDO DE CENTENO } \\
\text { - ALMIDÓN } \\
\text { AGLUTINANTE GRASO } \\
\text { - ACEITE SECATIVO } \\
\text { - ACEITE DE LINAZA } \\
\text { - ACEITE DE NUEZ }\end{array}$ \\
\hline $\begin{array}{l}\text { SUSTANCIA DE CARGA/RELLENO: } \\
\text { - SULFATO DE CALCIO } \\
\text { - CARBONATO DE CALCIO }\end{array}$ & $\begin{array}{l}\text { SUSTANCIA DE CARGA/RELLENO } \\
\text { - SULFATO DE CALCIO } \\
\text { - CARBONATO DE CALCIO }\end{array}$ \\
\hline $\begin{array}{l}\text { COLORES CUBRIENTES: } \\
\text { - BLANCO DE PLOMO } \\
\text { - TIERRAS (OCRE, ROJO, PARDA) }\end{array}$ & $\begin{array}{l}\text { COLORES CUBRIENTES: } \\
\text { - BLANCOS: } \\
\text { - BLANCO DE PLOMO } \\
\text { - LITOPÓN } \\
\text { - TIERRAS (OCRE, ROJO, PARDA) } \\
\text { - NEGRO }\end{array}$ \\
\hline $\begin{array}{l}\text { AISLANTE: } \\
\text { - COLAS ANIMALES } \\
\text { - ACEITES SECATIVOS }\end{array}$ & $\begin{array}{l}\text { AISLANTE: } \\
\text { • COLAS ANIMALES }\end{array}$ \\
\hline $\begin{array}{l}\text { ADITIVOS: } \\
\text { - POLVO DE LADRILLO }\end{array}$ & $\begin{array}{l}\text { ADITIVOS: } \\
\text { - CENIZA } \\
\text { - HARINA } \\
\text { - AZÚCAR }\end{array}$ \\
\hline
\end{tabular}

Cuadro $\mathrm{N}^{\circ}$ 3: Tipos de colas

\begin{tabular}{|c|c|c|c|}
\hline TIPO DE COLA & TRATADO & SOPORTE & TÉCNICA \\
\hline $\begin{array}{l}\text { Piel y cuerno de ciervo } \\
\text { Pergamino } \\
\text { Pescado } \\
\text { Piel de cabrito } \\
\text { Hueso o cuerno de ciervo } \\
\text { Piel de guante } \\
\text { Piel de carnero } \\
\text { Piel de carnero } \\
\text { Piel de cerdo } \\
\text { Cola de ajo }\end{array}$ & $\begin{array}{l}\text { Teófilo } \\
\text { Heraclius } \\
\text { Cennini } \\
\text { Cennini } \\
\text { J. Begue } \\
\text { T. Mayerne } \\
\text { Pacheco } \\
\text { Palomino } \\
\text { Volpato } \\
\text { Pacheco }\end{array}$ & $\begin{array}{l}\text { Madera } \\
\text { Madera } \\
\text { Madera } \\
\text { Madera } \\
\text { Madera } \\
\text { Madera } \\
\text { Madera } \\
\text { Madera } \\
\text { Madera } \\
\text { Madera }\end{array}$ & $\begin{array}{l}\text { Temple } \\
\text { Temple } \\
\text { Temple } \\
\text { Temple } \\
\text { Temple } \\
\text { Temple } \\
\text { Temple } \\
\text { Temple } \\
\text { Oleo } \\
\text { Temple }\end{array}$ \\
\hline $\begin{array}{l}\text { Almidón }\left({ }^{*}\right) \\
\text { Harina }\left(^{\star}\right) \\
\text { Harina }\left(^{* \star}\right) \\
\text { Harina }\left(^{* *}\right) \\
\text { Harina }\left(^{* \star}\right)\end{array}$ & $\begin{array}{l}\text { Cennini } \\
\text { Armenini } \\
\text { Vasari } \\
\text { Pacheco } \\
\text { Palomino }\end{array}$ & $\begin{array}{l}\text { Tela } \\
\text { Tela } \\
\text { Tela } \\
\text { Tela } \\
\text { Tela }\end{array}$ & $\begin{array}{l}\text { Temple } \\
\text { Temple } \\
\text { Oleo } \\
\text { Oleo } \\
\text { Oleo }\end{array}$ \\
\hline
\end{tabular}

Existen varios tipos de proteínas:

- Colágenos. Generalmente contenidas en las colas animales.

- Albúminas. Se encuentran fundamentalmente en los aglutinantes a base de huevo.

- Caseína. Se localizan en los derivados de productos lácteos. 
Cuadro $\mathrm{N}^{\circ}$ 4: Aglutinantes grasos

\begin{tabular}{l|l|l|l}
\hline ACEITE SECATIV0 & \multicolumn{1}{c}{ TRATADO } & SOPORTE & \multicolumn{1}{c}{ TÉCNICA } \\
\hline Aceite de linaza & Heraclius & Madera & ¿Oleo? \\
Aceite de linaza & Leonardo & Madera & Oleo \\
Aceite de linaza/nuez & Vasari & Madera & Oleo \\
Aceite de linaza & Pacheco & Madera & Oleo \\
Aceite de linaza & Felibien & Madera & Oleo \\
Aceite de linaza & Palomino & Madera & Oleo \\
\hline Aceite de linaza ( $\left.{ }^{*}\right)$ & Vasari & Tela & Oleo \\
Aceite de linaza $\left.{ }^{*}\right)$ & Armenini & Tela & Oleo \\
Aceite de linaza & Mayerne & Tela & Oleo \\
Aceite de linaza $\left.{ }^{*}\right)$ & Pacheco & Tela & Oleo \\
Aceite de linaza/nuez & Felibien & Tela & Oleo \\
Aceite de linaza & Palomino & Tela & Oleo \\
Aceite de linaza & Volpato & Tela & Oleo \\
\hline \multicolumn{2}{|c|}{$\left.{ }^{*}\right)=$ Aditivo en las imprimaciones de harina y pigmento. }
\end{tabular}

Dentro de este grupo hemos encontrado profusamente descritas en las fuentes consultadas las colas animales, generalmente cómo único aglutinante de las preparaciones magras.

Derivados vegetales. Las colas vegetales empleadas en estas capas son las compuestas básicamente por almidón y dextrina. El almidón se encuentra presente en la naturaleza en los granos, semillas, tallos, etc de las plantas, mientras que la dextrina se extrae del calentamiento en seco del almidón. De este grupo sólo hemos encontrado referencias sobre el empleo del almidón y de la harina. (Véase cuadro $n^{\circ} 3$ ).

Los aglutinantes grasos. Básicamente se han utilizado en la composición de la capa de imprimación, coloreada o no, con independencia de si intervienen de forma aislada -imprimación del soporte de tela para la técnica del óleo-, o de si forman parte de un fondo mixto para el soporte de madera - aplicada sobre una preparación magra con o sin capa aislante intermedia de cola animal o de aceite secativo-.

Tradicionalmente se han venido empleando en la composición de estas capas aquellos que se obtienen de las semillas, granos y frutos de ciertas plantas: aceite de lino y de nueces. Su empleo masivo se debe a la propiedad que tienen de formar una película homogénea transcurrido algunos días desde su aplicación en capa delgada. El más empleado y casi unánimemente descrito en las fuentes ha sido el aceite de linaza, sólo en Vasari y Felibien hemos encontrado referencias al uso del aceite de nueces como aglutinante de la capa de imprimación.

\section{Cargas y pigmentos}

En este apartado describiremos los principales materiales empleados en la capa de preparación e imprimación de los soportes pictóricos de madera y tela, como sustancias de carga y pigmentos.
Sustancias de carga: Son aquellas que tradicionalmente se han venido utilizando como colorantes y espesantes. Sus propiedades las hacen insustituibles en el estrato de preparación: son inertes o casi inertes, tienen gran poder cubriente aglutinadas en un medio acuoso magros y constituyen un estrato idóneo para aplicar la pintura.

Se han venido usando de forma habitual en la Historia de la Pintura con este fin el sulfato de calcio y el carbonato de calcio.

- Sulfato de calcio: Bajo esta denominación se agrupan un conjunto de materiales naturales y artificiales derivados del sulfato de calcio y de una cierta cantidad de agua de cristalización. En la Naturaleza se presenta bajo dos formas diferentes anhidrita (Ca SO4) y sulfato de calcio hidratado ( $\mathrm{Ca} \mathrm{So} 4+$ $\mathrm{H} 2 \mathrm{O}$ ). Ambos materiales se pueden modificar por calcinación, reduciendo a voluntad el agua de cristalización y dando como resultado modificaciones del producto original. Dentro de este grupo los más utilizados en la capa de preparación han sido los hemihidratos, obtenidos calentando el sulfato de calcio por encima de los $110^{\circ} \mathrm{C}$, esta reacción es reversible con la adición de agua, obteniéndose de esta forma sulfato de calcio bihidratado de estructura diferente del natural. Si se continua calentando el hemihidrato por encima de $190^{\circ} \mathrm{C}$. se obtiene el sulfato de calcio anhidrido.

- Carbonato de calcio: Se encuentra en la naturaleza bajo formas muy diversas, la más natural es la calcita (Ca CO3). Está presente en el reino animal, vegetal y mineral, como por ejemplo: en las conchas de algunos moluscos, en fósiles de origen reciente y en esqueletos de animales marinos como las foraminíferas y los "coccolithophoraidae". El carbonato de calcio también se manifiesta en las rocas sedimentarias, principalmente: creta, calizas, rocas metamórficas como el mármol, e incluso en las ígneas.

El carbonato de calcio es de color blanco muy puro por ello se ha empleado masivamente desde tiempos muy antiguos con fines artísticos. Dentro de este grupo cabe destacar la calcita y la creta.

La creta roca blanda porosa y frágil se presenta en la naturaleza estratificada en láminas en diversas partes de la superficie terrestre. La que deriva del limo marino presenta restos de fósiles en su composición, siendo los cocolitos los que más las caracteriza. Estos presentan un aspecto característico en forma de pequeños discos ovales o esféricos de aproximadamente de 3 a 8 micras de diámetro. Los cocolitos se encuentran en rocas calcáreas formadas en diferentes edades geológicas en depósitos sedimentarios.

También este material se encuentra en otras variedades orgánicas entre las que cabe destacar el blanco de concha, el blanco de coral y el blanco de cáscara de huevo.

Hemos encontrado numerosas referencia al uso de estos materiales en los tratados consultados, funda- 
mentalmente del sulfato de calcio empleado en la composición del soporte de madera, no obstante algunos de ellos han citado el uso del carbonato de calcio bien como carga de la preparación magra (Teofilo), como carga de la preparación en un fondo mixto (T. de Mayerné), o como carga en una imprimación oleosa (A. Felibién). Las conclusiones se exponen en el cuadro $n^{\circ} 5$

\section{Colores cubrientes}

Se han venido utilizando básicamente en la capa de imprimación ciertos pigmentos que por sus propiedades cubrientes, de tinción o secativas, contribuían a dejar apto el soporte pictórico para pintar. En las imprimaciones blancas el pigmento que de forma unánime han recomendado estos autores ha sido el blanco de plomo, mientras que en las coloreadas la gama de tierras, ocres rojas y pardas han sido las más ponderadas, mezcladas o no con blanco y negro para obtener la tonalidad deseada. (Véase cuadro $\mathrm{n}^{\circ}$ 6).

- Blanco de plomo: Es uno de los pigmentos blancos que se han empleado con mayor profusión con fines pictóricos en la historia de la Pintura hasta el siglo XIX. El blanco de plomo que se utiliza como pigmento es carbonato básico de plomo [2 Pb Co3. $\mathrm{Pb}(\mathrm{OH}) 2]$. Se ha fabricado artificialmente desde tiempo muy antiguos, siendo por ello, uno de los pigmentos sintéticos más antiguos que se conocen. Su uso como pigmento con fines pictóricos es mucho más apto en las técnicas grasas que en las magras.

- Ocres, tierras rojas y pardas: Son pigmentos naturales usados desde la Antigüedad, derivan de minerales y depósitos sedimentarios que se encuentran en la corteza terrestre. Están considerados como los más estables por su buen comportamiento y resistencia al medio ambiente y al aglutinante. Se comportan bien en todas las técnicas pictóricas y han sido profusamente empleados como pigmentos en las imprimaciones coloreadas que recibe el soporte de madera sobre una base magra aislada o no, o sobre el soporte de tela previamente aislado con cola.

Las fuentes consultadas nos citan a los siguientes:

- Ocres. Tierras naturales compuestas principalmente por silice y arcilla, con mayor o menor grado de impurezas que son eliminadas en curso de fabricación para obtener un pigmento estable. Su manufacturación es muy simple consiste básicamente en: extracción del mineral, selección, molido, lavado y decantado, quedando el producto resultante después de su secado apto para su uso.

- Tierras rojas. Se incluyen bajo esta denominación una amplia gama de pigmentos compuestos por óxido de hierro en sus dos formas, hidratada y sin hidratar. Se encuentran presentes en la Naturaleza en amplios depósitos de rocas ferruginosas que contienen un porcentaje variable de arcilla. Su tonalidad dependerá en gran medida del grado
Cuadro $N^{\circ}$ 5: Sustancias empleadas como cargas

\begin{tabular}{|c|c|c|c|}
\hline SUSTANCIA DE CARGA & TRATADO & SOPORTE & TÉCNICA \\
\hline $\begin{array}{l}\text { Sulfato de calcio } \\
\text { Sulfato de calcio } \\
\text { Sulfato de calcio }\left(^{\star}\right) \\
\text { Sulfato de calcio } \\
\text { Sulfato de calcio } \\
\text { Sulfato de calcio }\left(^{(\star}\right) \\
\text { Sulfato de calcio } \\
\text { Sulfato de calcio } \\
\text { Sulfato de calcio }\left(^{(\star}\right) \\
\text { Sulfato de calcio }\left(^{(\star *}\right) \\
\text { Sulfato de calcio }\end{array}$ & $\begin{array}{l}\text { Teofilo } \\
\text { Cennini } \\
\text { Cennini } \\
\text { J. Begue } \\
\text { J. Begue } \\
\text { Vasari } \\
\text { Armenini } \\
\text { Armenini } \\
\text { Armenini } \\
\text { Pacheco } \\
\text { Palomino }\end{array}$ & $\begin{array}{l}\text { Madera } \\
\text { Madera } \\
\text { Tela } \\
\text { Madera } \\
\text { Tela } \\
\text { Madera } \\
\text { Madera } \\
\text { Tela } \\
\text { Madera } \\
\text { Madera } \\
\text { Madera }\end{array}$ & $\begin{array}{l}\text { Temple } \\
\text { Temple } \\
\text { Temple } \\
\text { ¿? } \\
\text { ¿? } \\
\text { Oleo } \\
\text { Temple } \\
\text { Temple } \\
\text { Oleo } \\
\text { Oleo } \\
\text { Temple }\end{array}$ \\
\hline $\begin{array}{l}\text { Carbonato de calcio } \\
\left.\text { Carbonato de calcio }{ }^{\star \star}\right) \\
\text { Carbonato de calcio }\end{array}$ & $\begin{array}{l}\text { Teofilo } \\
\text { Mayerne } \\
\text { Felibien }\end{array}$ & $\begin{array}{l}\text { Madera } \\
\text { Madera } \\
\text { Tela }\end{array}$ & $\begin{array}{l}\text { Temple } \\
\text { Oleo } \\
\text { Oleo }\end{array}$ \\
\hline \multicolumn{4}{|c|}{$\begin{array}{l}\left(^{*}\right)=\text { Como componente de la capa de preparación magra conjuntamente con } \\
\text { el almidón y el azúcar. } \\
(* *)=\text { Componente de la preparación magra en un fondo mixto. }\end{array}$} \\
\hline
\end{tabular}

de hidratación, de la manufacturación y del tamaño del grano principalmente. Las variedades más hidratadas son de tonalidad rojo oscuro y las menos hidratadas abarcan matices que van del rojo al amarillo. Su empleo es apto en todas las técnicas, si bien aglutinados en aceite secativo son de lento secado.

- Tierras pardas. Como en el caso anterior abarca una extensa gama de pigmentos compuestos básicamente por arcillas y óxido de hierro, además de otros componentes que determinarán su composición: Tierra de Siena natural (ocre con ácido silícico y óxido de hierro), Tierra de Siena Tostada (idéntica composición, la tonalidad se obtiene por calcinación), Sombra Natural (ocre con un porcentaje de hierro y manganeso) y la sombra Tostada (se obtiene por calcinación de la anterior). Los más utilizados en la capa de imprimación son los mencionados anteriormente, no obstante cabe decir, que el empleo de la tierra de Siena y la Tierra de Sombra Natural no están muy indicadas en técnicas oleosa por su lento secado y rápido oscurecimiento.

- Negros. Los más usados en Pintura desde tiempo que se remontan a la Antigüedad provienen fundamentalmente de la calcinación, bien de huesos (negro de hueso, marfil), de troncos vegetales (negro de vid) y de la recogida del humo que se produce durante la carbonización (negro de lámpara). Son pigmentos que se caracterizan por ser muy estables y adaptables a todas las técnicas pictóricas. Los más empleados en la capa de imprimación han sido los siguientes:

- Negro de hueso. Fabricado a partir de huesos de animales, el más estimado por su color intenso es el obtenido por la calcinación del marfil y conocido bajo el mismo nombre. En su composición se detecta carbón, fosfato de calcio, carbonato de calcio y ceniza principalmente. 
- Negro de carbón. Se obtiene a partir del humo que se desprende de la calcinación de la madera, el pro- ducto resultante es carbón casi puro. Sus partículas son muy pequeñas y homogéneas, de ligero matiz azulado y poco poder cubriente .

\section{Cuadro $N^{\circ}$ 6: Colores cubrientes}

\begin{tabular}{|c|c|c|c|}
\hline COLORES CUBRIENTES & TRATADO & SOPORTE & TÉCNICA \\
\hline $\begin{array}{l}\text { Blanco de plomo } \\
\text { Blanco de plomo }\left(^{*}\right) \\
\text { Blanco de plomo }\left(^{* *}\right) \\
\text { Blanco de plomo }\left(^{*}\right) \\
\text { Blanco de plomo }\left(^{(*}\right) \\
\text { Blanco de plomo } \\
\text { Blanco de plomo }\left(^{\star}\right) \\
\text { Blanco de plomo }\left(^{*}\right) \\
\text { Blanco de plomo }\left(^{*}\right) \\
\text { Blanco de plomo }\left(^{*}\right)\end{array}$ & $\begin{array}{l}\text { Heraclius } \\
\text { Vasari } \\
\text { Vasari } \\
\text { Armenini } \\
\text { Armenini } \\
\text { Mayerne } \\
\text { Mayerne } \\
\text { Pacheco } \\
\text { Pacheco } \\
\text { Felibien }\end{array}$ & $\begin{array}{l}\text { Madera } \\
\text { Madera } \\
\text { Tela } \\
\text { Madera } \\
\text { Tela } \\
\text { Madera } \\
\text { Tela } \\
\text { Madera } \\
\text { Tela } \\
\text { Tela }\end{array}$ & $\begin{array}{l}\text { Oleo } \\
\text { Oleo } \\
\text { Oleo } \\
\text { Oleo } \\
\text { Oleo } \\
\text { Oleo } \\
\text { Oleo } \\
\text { Oleo } \\
\text { Oleo } \\
\text { Oleo }\end{array}$ \\
\hline $\begin{array}{l}\text { Tierra de Campana } \\
\text { Sombra u ocre y blanco } \\
\text { Bol, sombra y blanco } \\
\text { Ocre, blanco y negro } \\
\text { Sombra y blanco } \\
\text { Tierras rojas y negro } \\
\text { Tierras, ocre rojo } \\
\text { y carbonato de calcio } \\
\text { Tierras, blanco y negro } \\
\text { Tierras } \\
\text { Tierra roja y sombra }\end{array}$ & $\begin{array}{l}\text { Vasari } \\
\text { Armenini } \\
\text { Mayerne } \\
\text { Mayerne } \\
\text { Pacheco } \\
\text { Pacheco } \\
\text { Felibien } \\
\text { Felibien } \\
\text { Palomino } \\
\text { Volpato }\end{array}$ & $\begin{array}{l}\text { Madera } \\
\text { Madera } \\
\text { Tela } \\
\text { Tela } \\
\text { Madera } \\
\text { Tela } \\
\\
\text { Madera } \\
\text { Tela } \\
\text { Tela } \\
\text { Tela }\end{array}$ & $\begin{array}{l}\text { Oleo } \\
\text { Oleo } \\
\text { Oleo } \\
\text { Oleo } \\
\text { Oleo } \\
\text { Oleo } \\
\\
\text { Oleo } \\
\text { Oleo } \\
\text { Oleo } \\
\text { Oleo }\end{array}$ \\
\hline
\end{tabular}

\section{CONCLUSIONES}

Como hemos visto hasta el momento los autores consultados establecen una clara distinción entre la composición material de la preparación y de la imprimación en función de la técnica pictórica y el soporte. Este hecho se hace particularmente notable en el periodo de transición entre el soporte de madera y el desarrollo de la técnica del óleo en detrimento del temple, durante el cual conviven conjuntamente la preparación magra y la imprimación oleosa y aparece la capa aislante entre ellos.

Generalmente se recomienda que la composición de esto/s estrato/s sean de la misma naturaleza que la de la técnica seleccionada por el artista.

Y por último, la gran mayoría de ellos coinciden en señalar la importancia que tiene para la buena conservación de la pintura acabada, adecuar y preparar convenientemente el soporte.

En el cuadro $n^{\circ} 7$ se resume la naturaleza y evolución de las capas que estudiamos desde la visión de los tratados consultados.

\section{Notas}

I. T. Rousseau, H. Von Sonnenburg, "La preparation des peintures". Reunion du Comité de I'Icom pour les Laboratoires de Musées et du Sous Comité de l'Icom pour le traitements des peintures. Leningrad-Moscou, september (1963), p: 2-3.

2. Siempre en la realización de estas capas se tiene por correcto respetar la norma de graso sobre magro, si se quiere evitar alteraciones prematuras que dañarían estéticamente e irrreversiblemente la conservación natural de la pintura.

3. B. Palmer "The technology, examination et conservation of the Fayum portraits in the Petrie Museum". Studies in Conservation, vol 24, (1979), p: I- 13
4. N. Goetghebeur "Examen et traitement d'un sarcophage égyptien du VI eeshui à Anvers, Bulletin I.R.P.A., vol XI (1969), p: $59-65$.

J. Natchinkina, E. Cheinina "Conservation d'objets de l'Egipte ancienne en bois polychrome au Museé de l' Ermitage d' Etat". Icom Preprints, Otawa ( 199 |), p: I-8

5. M. P. Merrifield "Original tratises on the arts of painting" Dover Publications Inc. New York. (1967), vol I, p: 228

6. G. Vasari "Vasari on technique": Dover Publications Inc. New York (1960), p: 224 y ss.

\section{Bibliografía}

G. B. ARMENINI Dé veri precetti della Pittura. Giulio Einaudi Editore. Torino (1988)

CENNINO CENNINI

Tratado de la pintura. Manuales Meseguer. Ed.: Sucesor de E. Meseguer, $4^{\mathrm{a}}$ ed. (1979)

Il Libro dell'arte o Trattato della Pittura a cura di Fernando Tempesti. Ed.: Longanesi \& C. Milano (1975).

L. DA VINCI Tratado de Pintura. Editora Nacional, $2^{\circ}$ ed.

A. FELIBIEN Des principes de l'Achitecture, de la Sculpture, de la Peinture et des autres Arts qui en dépendent. $1^{\circ}$ ed Published Paris (1969). Republished by Gregg Press Limited England.

Ma JOSÉ. GONZÁLEZ LÓPEZ . Estudio de las preparaciones de pintura sobre soportes de tela y tabla. Caracterización de sus principales componentes, comportamiento y factor de deterioro. 1993. Editorial de la Universidad de Sevilla. ISBN: 84-472-0 I05-8.
T. DE MAYERNE Pictorja Scultorja \& quae subalternarum artium. Le manuscrit de Turquet de Mayerne. Audin Lyon (1974).

M. P. MERRIFIELD. Original tratises on the arts of painting. Dover Publications Inc. New York. (1967), 2 vol.

F. PACHECO. Arte de la Pintura. Ed. Leda, $2^{a}$ ed. (1982).

A. PALOMINO. El Museo Pictórico y escala óptica. El Parnaso español pintoresco y laureado. Ed.: Aguilar S.A. (1988), 3 vol.

THEOPHILUS. On divers arts. Ed Dover Publications Inc. New York. (1979).

G. VASARI VASARI ON TECHNIQUE. Dover Publications Inc. New York (1960), p: 224 y ss. 
Cuadro $N^{\circ} 7$ : Naturaleza y evolución de la capa de preparación

\begin{tabular}{|c|c|c|c|c|c|c|c|c|c|}
\hline \multirow[t]{2}{*}{ SIGLO } & \multirow[t]{2}{*}{ TRATADISTA } & \multicolumn{3}{|c|}{ SOPORTE } & \multicolumn{4}{|c|}{ PREPARACIÓN } & \multirow[t]{2}{*}{ TÉCNICA } \\
\hline & & Madera & Mixto & Tela & Aislante & Preparación & Aislante & Imprimación & \\
\hline XII? & Teofilo & $\bullet$ & • & - & - & Magra & $\bullet$ & - & Temple \\
\hline$X \mid I$ & Heraclio & $\bullet$ & $\begin{array}{l}\bullet \\
-\end{array}$ & - & Cola & $\begin{array}{l}- \\
-\end{array}$ & $\begin{array}{l}- \\
-\end{array}$ & $\begin{array}{c}\text { Blanca } \\
-\end{array}$ & $\begin{array}{l}\text { ¿Oleo? } \\
\text { Temple }\end{array}$ \\
\hline$X V$ & J. Begue & $\bullet$ & - & - & Cola & Magra & - & - & $i ?$ \\
\hline$X V$ & C. Cennini & $\begin{array}{l}\bullet \\
- \\
-\end{array}$ & $\begin{array}{l}\bullet \\
- \\
-\end{array}$ & - & $\begin{array}{l}\text { Cola } \\
\text { Cola } \\
\text { Cola }\end{array}$ & $\begin{array}{l}\text { Magra } \\
\text { Magra } \\
-\end{array}$ & - & $\begin{array}{l}- \\
- \\
-\end{array}$ & $\begin{array}{l}\text { Temple } \\
\text { Temple } \\
\text { Temple }\end{array}$ \\
\hline$X V$ & L. Da Vinci & $\begin{array}{l}\bullet \\
-\end{array}$ & - & - & Cola & - & - & $\begin{array}{c}\text { Coloreada } \\
-\end{array}$ & $\begin{array}{l}\text { Oleo } \\
\text { Temple }\end{array}$ \\
\hline$X V I$ & Vasari & $\begin{array}{l}\bullet \\
\bullet \\
\bullet \\
- \\
-\end{array}$ & $\begin{array}{l}- \\
- \\
- \\
- \\
-\end{array}$ & $\begin{array}{l}- \\
- \\
- \\
- \\
\bullet\end{array}$ & $\begin{array}{c}- \\
\text { Cola } \\
- \\
\text { Cola } \\
\text { Cola }\end{array}$ & $\begin{array}{c}\text { Magra } \\
- \\
\text { Magra } \\
- \\
\text { Magra }\end{array}$ & $\begin{array}{c}- \\
- \\
- \\
- \\
\text { Cola }\end{array}$ & $\begin{array}{c}- \\
- \\
\text { Cola } \\
\text { Harina } \\
\text { Coloreada }\end{array}$ & $\begin{array}{l}\text { Temple } \\
\text { Temple } \\
\text { Oleo } \\
\text { Oleo } \\
\text { Oleo }\end{array}$ \\
\hline$X V I$ & Armenini & $\begin{array}{l}\bullet \\
\bullet \\
- \\
- \\
- \\
-\end{array}$ & $\begin{array}{l}- \\
- \\
- \\
- \\
- \\
-\end{array}$ & $\begin{array}{l}- \\
- \\
\dot{ } \\
\dot{ } \\
\dot{ }\end{array}$ & $\begin{array}{c}- \\
\text { Cola } \\
\text { Cola } \\
- \\
\text { Cola } \\
-\end{array}$ & $\begin{array}{c}\text { Magra } \\
\text { Magra } \\
- \\
\text { Magra } \\
- \\
-\end{array}$ & $\begin{array}{c}- \\
- \\
- \\
- \\
- \\
\text { Cola }\end{array}$ & $\begin{array}{c}- \\
\text { Coloreada } \\
- \\
- \\
\text { Coloreada } \\
\text { Doble }\end{array}$ & $\begin{array}{c}\text { Temple } \\
\text { Emulsión } \\
\text { Temple } \\
\text { Temple } \\
\text { Oleo } \\
\text { Oleo }\end{array}$ \\
\hline$X V I I$ & T. Mayerne & - & - & - & Cola & $\begin{array}{l}\text { Magra } \\
\text { Magra }\end{array}$ & - & $\begin{array}{l}\text { Blanca } \\
\text { Coloreada }\end{array}$ & $\begin{array}{l}\text { Oleo } \\
\text { Oleo }\end{array}$ \\
\hline$X V I I$ & F. Pacheco & $\begin{array}{l}\bullet \\
\bullet \\
- \\
- \\
- \\
-\end{array}$ & $\begin{array}{l}- \\
- \\
- \\
- \\
- \\
-\end{array}$ & $\begin{array}{l}- \\
\dot{ } \\
\dot{ } \\
\dot{ } \\
\bullet\end{array}$ & $\begin{array}{c}- \\
\text { Cola } \\
\text { Cola } \\
- \\
\text { Cola } \\
\text { Cola }\end{array}$ & $\begin{array}{c}\text { Magra } \\
\text { Magra } \\
- \\
- \\
\text { Magra } \\
-\end{array}$ & $\begin{array}{l}- \\
- \\
- \\
- \\
- \\
-\end{array}$ & $\begin{array}{c}- \\
\text { Coloreada } \\
- \\
\text { Harina } \\
\text { Coloreada } \\
\text { Coloreada }\end{array}$ & $\begin{array}{c}\text { Temple } \\
\text { Oleo } \\
\text { Temple } \\
\text { Oleo } \\
\text { Oleo } \\
\text { Oleo }\end{array}$ \\
\hline$X V I I$ & Felibien & $\begin{array}{l}\bullet \\
- \\
- \\
-\end{array}$ & $\begin{array}{l}- \\
- \\
- \\
-\end{array}$ & - & $\begin{array}{c}- \\
\text { Cola } \\
- \\
\text { Cola }\end{array}$ & $\begin{array}{c}\text { Magra } \\
\text { Magra } \\
\text { Magra } \\
-\end{array}$ & $\begin{array}{l}- \\
- \\
- \\
-\end{array}$ & $\begin{array}{c}- \\
\text { Coloreada } \\
- \\
\text { Coloreada }\end{array}$ & $\begin{array}{l}\text { Temple } \\
\text { Oleo } \\
\text { Temple } \\
\text { Oleo }\end{array}$ \\
\hline XVIII & Palomino & $\begin{array}{l}\dot{\bullet} \\
\bullet \\
- \\
- \\
-\end{array}$ & $\begin{array}{l}- \\
- \\
- \\
- \\
- \\
-\end{array}$ & $\begin{array}{l}\bullet \\
- \\
- \\
- \\
\bullet \\
\bullet\end{array}$ & $\begin{array}{c}\text { Cola } \\
- \\
\text { Cola } \\
\text { Cola } \\
- \\
\text { Cola }\end{array}$ & $\begin{array}{c}\text { Magra } \\
\text { Magra } \\
\text { Magra } \\
\text { Magra } \\
- \\
-\end{array}$ & $\begin{array}{c}\text { Cola } \\
- \\
- \\
- \\
- \\
-\end{array}$ & $\begin{array}{c}- \\
\text { Coloreada } \\
\text { Coloreada } \\
- \\
\text { Doble } \\
\text { Coloreada }\end{array}$ & $\begin{array}{l}\text { Temple } \\
\text { Oleo } \\
\text { Oleo } \\
\text { Temple } \\
\text { Oleo } \\
\text { Oleo }\end{array}$ \\
\hline XVIII & Volpato & $\begin{array}{l}- \\
-\end{array}$ & $\begin{array}{l}- \\
-\end{array}$ & • & $\begin{array}{c}\text { Cola } \\
-\end{array}$ & $\begin{array}{l}- \\
-\end{array}$ & $\begin{array}{l}- \\
-\end{array}$ & $\begin{array}{c}- \\
\text { Coloreada }\end{array}$ & $\begin{array}{l}\text { Temple } \\
\text { Oleo }\end{array}$ \\
\hline
\end{tabular}

